\title{
An overview of the British Columbia Glomerulonephritis network and registry: integrating knowledge generation and translation within a single framework
}

Sean Barbour ${ }^{1,2,3^{*}}$, Monica Beaulieu ${ }^{1,2}$, Jagbir Gill ${ }^{1,2,3}$, Ognjenka Djurdjev², Heather Reich ${ }^{4}$ and Adeera Levin ${ }^{1,2,3}$

\begin{abstract}
Background: Glomerulonephritis (GN) is a group of rare kidney diseases with a substantial health burden and high risk of progression to end-stage renal disease. Research in GN has been limited by poor availability of large comprehensive registries. Substantial variations in access to and administration of treatment and outcomes in GN have been described. Leveraging provincial resources and existing infrastructure, the British Columbia (BC) GN Network is an initiative which serves to combine research and clinical care objectives. The goal of the BC GN Network is to coordinate and improve health care, including robust data capture, on all patients with GN in BC, a Canadian province of over 4.6 million people. This provincial initiative will serve as a model for Canadian or other national and international endeavours.
\end{abstract}

Description: The BC Provincial Renal Agency (BCPRA) is the provincial governmental agency responsible for health delivery for all kidney patients in BC. The BC GN Network has been created by the BCPRA to ensure high quality and equitable access to care for all patients with GN and is a platform for evidence based clinical care programs and associated health policy. All patients with biopsy-proven GN are registered at the time of kidney biopsy into the BCPRA provincial database of kidney disease patients, forming the BC GN Registry. Thereafter, all laboratory results and renal related outcomes are captured automatically. Histology data and core clinical variables are entered into the database. Additional linkages between the GN Registry and administrative databases ensure robust capture of medications, hospital admissions, health care utilization, comorbidities, cancer and cardiac outcomes, and vital statistics.

Conclusions: The BC GN Network and Registry is a unique model in that it combines robust data capture, data linkages, and health care delivery and evaluation into one integrated system. This model utilizes existing health infrastructure to prospectively capture population level data on patients with $G N$, producing a rich dataset capable of real-time identification and evaluation of GN health policy initiatives, of supporting observational cohort studies and health services research in GN, and of facilitating patient recruitment into GN clinical trials.

Keywords: Glomerulonephritis, Registry, Database, Health services, Knowledge translation, British Columbia, Canada

\footnotetext{
* Correspondence: Sean.Barbour@vch.ca

'Division of Nephrology, University of British Columbia, Vancouver, BC,

Canada

${ }^{2}$ BC Provincial Renal Agency, Vancouver, BC, Canada

Full list of author information is available at the end of the article
} 


\section{Background}

While glomerulonephritis (GN) is considered a group of rare diseases, with an incidence of 0.7-2.8 per 100000/ year depending on the specific type of GN, collectively they may consume substantial health care resources $[1,2]$. The risk of progression to end-stage renal disease (ESRD) is high, between $20-50 \%$, such that GN is the second most common cause of ESRD in Canada [3-9]. Further, there is additional morbidity and cost associated with the treatment of $\mathrm{GN}$, related both to expensive immunosuppressive medications, and infectious, malignancy or other complications of treatment. Consequently, the burden of GN to the health care system is substantial.

Due to the rarity of GN, registries that sample large geographic regions, and thus large numbers of patients, are required for collection of sufficient data to evaluate and improve health policy and systems of care for GN patients, and to facilitate clinic outcomes research in glomerular diseases $[1,10]$. The infrequent nature of the disease contributes to the poor understanding of these conditions by individual clinicians who may be exposed to small numbers of patients. Similar to cancer registries, the potential benefits of central registration of GN patients into regional databases may include enhanced physician collaboration resulting in improved patient care [1]. The absence of such registries significantly limits the understanding of the natural history of GN and the recruitment of GN patients into clinical trials, and thus impedes research and results in a void of evidencebased treatment recommendations. While existing GN registries have substantially contributed to the advancement of GN research, they are often research-based databases limited in their recruitment scope, expensive and labour-intensive to maintain, and not well integrated into patient care. Given these limitations they are not likely to be sustainable in the long-term.

The British Columbia (BC) GN Network and Registry has been created to address the limitations of other registries by leveraging existing health services infrastructure and data capture to facilitate and coordinate the care of all patients with $\mathrm{GN}$ in $\mathrm{BC}$, a Canadian province of over 4.6 million people with a universal health care system. The components of the BC GN Network include the creation of a formal GN Registry embedded within an existing provincial information system, and the creation of an advisory group of relevant stakeholders including physicians, researchers, pharmacists, nurses and analysts, with direct responsibility for the administration of the BC GN Network and accountability for its outcomes. Both the database and the advisory group utilize existing infrastructure and processes within a larger provincial kidney disease network.

\section{Overarching goals of the BC GN Network}

1. To create a sustainable framework for knowledge translation, policy development and implementation of best practice management through the systematic collection and analysis of data

2. To identify, implement and evaluate clinical care programs and health policy initiatives that improve the care of patients with GN in BC in a financially sustainable fashion.

3. To promote clinical outcomes, health policy and health services research in the field of GN.

4. To ensure knowledge translation of such initiatives to key stakeholders, including patients and physicians.

\section{Specific goals of the BC GN Registry}

1. To prospectively capture at the time of kidney biopsy all relevant clinical, laboratory, pathology and outcome data in GN patients.

2. To collate data regarding the incidence, prevalence, outcomes, and health care utilization of patients with GN.

3. To capture such data using a sustainable infrastructure that leverages existing administrative resources.

4. To ensure high quality data is available to identify, develop and evaluate new clinical care and health policy initiatives targeting patients with GN.

5. To provide a rich dataset capable of supporting clinical outcomes and health services research in the field of GN.

\section{Construction and content}

The provincial infrastructure for chronic kidney disease in $B C$

The BC Provincial Renal Agency (BCPRA) is a governmental organization that coordinates, monitors and funds the delivery of care to patients with kidney disease in BC (www.bcrenalagency.ca). The mandate of the BCPRA is to improve the quality of life and outcomes for patients with renal disease, and to promote a fiscally sustainable health care system. The BCPRA operates the Patient Records and Outcomes Management Information System (PROMIS), which is a provincial database of all patients with chronic kidney disease (CKD) in BC. PROMIS is an Oracle-based web-accessible database with over 10000 patient-records that was initiated in 2000 and captures patients with CKD, defined as evidence of kidney damage either with an estimated (e)GFR $<60 \mathrm{ml} / \mathrm{min} /$ $1.73 \mathrm{~m}^{2}$, or structural abnormalities on imaging or kidney biopsy. Registration in PROMIS is mandatory for funding of CKD medications (such as erythropoietin stimulating 
agents, phosphate binders or vitamin $\mathrm{D}$ analogues), access to multidisciplinary care teams, or to receive renal replacement therapy including dialysis and transplantation. As such, PROMIS serves as a central source of data for all patients seen by nephrologists or kidney care teams in BC. PROMIS interfaces with all laboratory systems in the province, which permits automatic data capture and upload into a central database. Clinical data captured in PROMIS includes comorbidities, medications, blood pressure, weight and height. Funding is in place for data capture and formal data quality checks.

While technically able to register any patient with GN, PROMIS was not specifically designed as a database for patients with glomerular diseases who often have normal eGFR. Review of existing biopsy databases identified that approximately $66 \%$ of patients with IgA nephropathy and $25 \%$ of those with pauci-immune glomerulonephritis are not registered in PROMIS, which may be related to preserved renal function in a subgroup of patients, or a competing risk of death preventing registration [11]. Further, GN patients in $\mathrm{BC}$ have historically been registered late in the course of their disease when renal function has significantly declined, with little historical data available between the time of kidney biopsy and the development of compromised kidney function requiring access to "CKD resources". The BC GN Network and Registry utilizes the existing infrastructure at both the BCPRA and PROMIS to capture data on patients with biopsy-proven $\mathrm{GN}$ as of the time of kidney biopsy.

\section{The structure of the BC GN Network}

The BC GN Network is an initiative of the BCPRA that coordinates and evaluates the care of patients with GN in BC, with 5 geographical health authorities with more than 70 nephrologists. The BC GN Network has an established set of terms of reference (see Additional file 1), and is comprised of a formal steering committee of stakeholders interested in GN, including physicians, researchers, pharmacists, nurses and data analysts. To maintain integration within the larger provincial renal community, the steering committee has reporting obligations to the nephrology research unit within the University of British Columbia and to administrative committees within the BCPRA, including the Executive Committee, the Medical Advisory Committee, the Pharmacy and Formulary Committee, and the Information Management Council.

The BC GN Network focuses on several domains of GN-related health care as outlined in Table 1. This includes developing and evaluating health policy in GN, encouraging clinical and health services research in glomerular diseases, engaging key stakeholders of the renal community, and improving education and knowledge translation to end users. In addition, a significant activity of the BC GN Network is the implementation and oversight of the $\mathrm{BC}$ GN Registry to collect data necessary to support the goals of the BC GN Network. Detailed information can be found on the website: www. bcrenalagency.ca/professionals/GNNetworkRegistry.

Table 1 Domains and activities of the BC GN Network with specific examples

\begin{tabular}{ll}
\hline Domains of the BC GN network & Examples \\
\hline Developing and evaluating health care initiatives specific to GN patients & GN specialty multi-disciplinary clinics with telehealth outreach to all \\
& geographic regions in the province \\
& $\begin{array}{l}\text { Standardized GN-specific immunosuppression orders and laboratory } \\
\text { requisitions } \\
\text { GN-specific laboratory and medication flow sheets } \\
\text { Collection of data necessary to support the goals of the BC GN Network }\end{array}$ \\
& Centralized funding for immunosuppression medications
\end{tabular}

Regular reporting to the provincial renal community on the incidence, prevalence, outcomes and health care utilization of GN patients in BC

Encouraging research in the field of GN

Utilization of the BC GN Registry for health outcomes and health services research

Facilitating recruitment of patients into $\mathrm{GN}$ clinical trials by identifying eligible patients in the BC GN Registry

Engaging patients, physicians and other members of the renal community in the development of GN-specific initiatives

BC GN Network Steering Committee has representation from multiple health care domains, including pharmacists, academic and community physicians, and database / analytic specialists

Education and knowledge translation to physicians, patients

Provincial GN rounds and other stakeholders

Patient education tools, ex. medication information sheets 


\section{The BC GN Registry}

As of March $1^{\text {st }} 2013$, the BC GN Registry prospectively captures data on all patients with $\mathrm{GN}$ in $\mathrm{BC}$ as of the time of kidney biopsy, with data storage in PROMIS. It is comprised of a combination of existing BCPRA resources, a new pathology database initiative, data extraction of key clinical variables, and the ability to link with administrative datasets to capture outcomes of interest (see Figure 1). The structure of the BC GN Network and Registry is shown in Figure 2.

\section{Integration of provincial and regional pathology databases into PROMIS}

All renal biopsies in $\mathrm{BC}$ are interpreted at two centres: the Royal Jubilee Hospital in Victoria, which processes all biopsies performed on Vancouver Island, and the BC Renal Pathology Laboratory based in Vancouver, which processes all biopsies in the remainder of the province. Prior to the 2013 launch of this initiative, each centre previously maintained independent locally managed pathology databases. These have been replaced with a single centralized pathology database within PROMIS containing key histologic variables from each biopsy performed (see Table 2). During the initial phase of the BC GN Network, histologic data from the pathology reports is being manually entered into PROMIS by trained data analysts. Development of a robust user-interface is ongoing and will permit pathologists to enter data directly at the time of biopsy interpretation, reducing duplication of data entry and ensuring seamless access to key data.

\section{Registration in the $B C$ GN Registry}

The inclusion criterion of the BC GN Registry is the diagnosis on renal biopsy of any glomerular disease found in Table 3. The diagnosis of GN results in automatic registration in PROMIS and the BC GN Registry, which triggers a set of activities that ensure consistent capture of essential data. Patients with other types of biopsy-proven renal diseases, such as diabetes, hypertensive nephrosclerosis or interstitial nephritis are not included in the GN registry. These patients are however captured in the provincial pathology database.

\section{Anticipated incidence of $G N$ in $B C$}

A review of existing pathology databases permits an estimate of the anticipated yearly incidence of GN in BC. During the 12-year prior from 2000-2012, there were 3473 cases of the common glomerular diseases, with an average of 289 cases per year (see Table 4). The most frequent types of GN in BC are lupus nephritis (62 cases per year), IgA nephropathy (69 cases per year), and focal segmental glomeruloslerosis (70 cases per year).

\section{Capture of full laboratory data}

Because the BC GN Registry is embedded within PROMIS, once the patient is registered all laboratory results are automatically uploaded through established and sanctioned interfaces between PROMIS and provincial laboratory systems (see above). Relevant laboratory results for patients with glomerular diseases, such as proteinuria, albumin, cholesterol, creatinine, and autoimmune and infectious disease serology are captured at whatever frequency they are ordered by the primary physician.

As part of a province-wide eGFR reporting initiative, creatinine measurements at all laboratories in $\mathrm{BC}$ have been calibrated using an isotope dilution mass spectrometry (IDMS) reference standard and used in the IDMS traceable version of the MDRD formula [13]. As such, all creatinine measurements and eGFR estimates are standardized and directly comparable across laboratories.

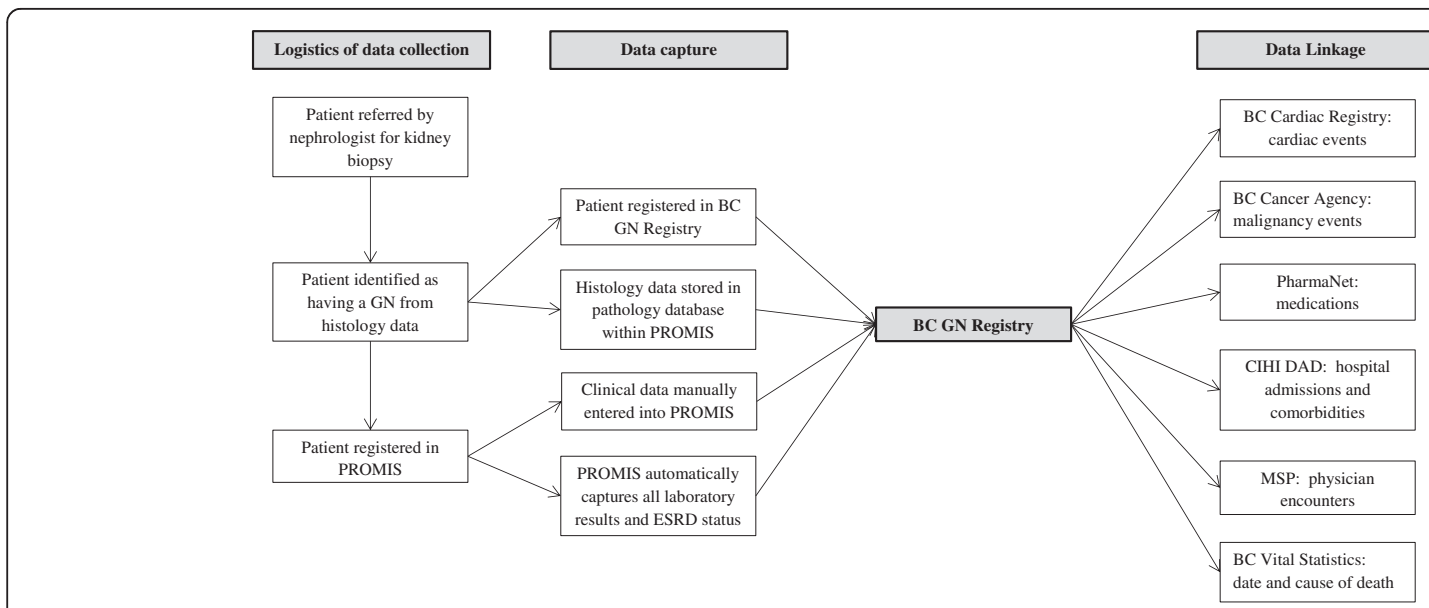

Figure 1 The logistics of data capture for the BC GN Registry. BCPRA = BC Provincial Renal Agency. CIHI DAD: Canadian Institute of Health Information Discharge Abstract Database. MSP = Medical Services Plan. 


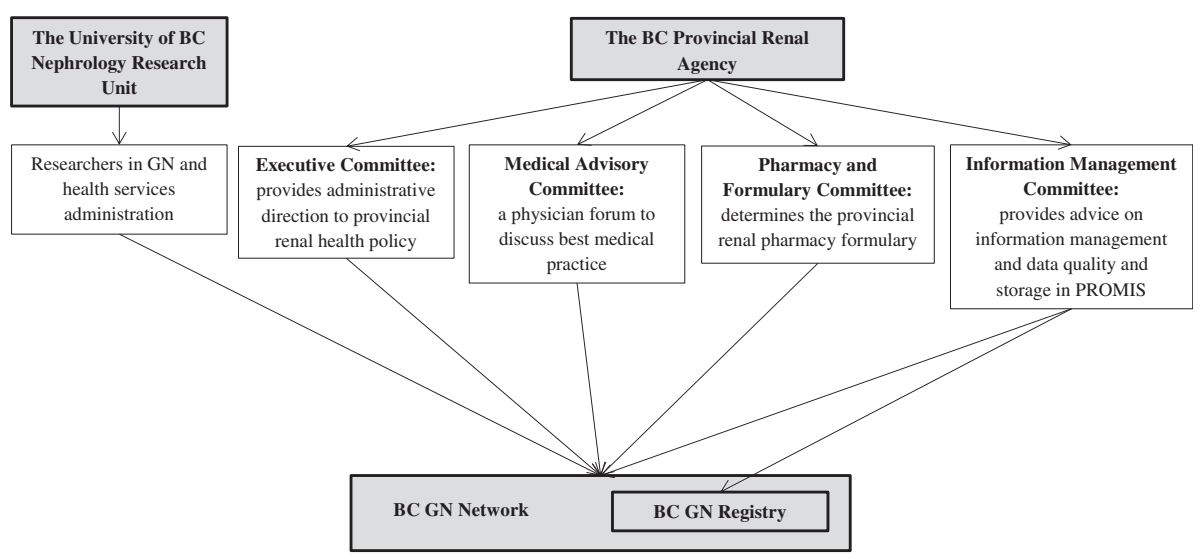

Figure 2 The structure of the BC GN Network and Registry with the University of British Columbia and the BC Provincial Renal Agency.

\section{Capture of clinical data}

Date of birth, sex, and geographic location are required at the time of registration in PROMIS. Additional clinical data is captured from the primary nephrologists in accordance with best practices and existing technologies. The clinical variables that are collected are as follows: comorbidities, medications, blood pressure, height and

Table 2 Variables stored in PROMIS as part of the provincial pathology database, with representative examples

\begin{tabular}{ll}
\hline $\begin{array}{l}\text { Variables in pathology } \\
\text { database }\end{array}$ & Examples \\
\hline Patient details & $\begin{array}{l}\text { Name, date of birth, Personal Health } \\
\text { Number, geographic location, sex }\end{array}$ \\
Biopsy details & $\begin{array}{l}\text { Date of biopsy, native versus transplant } \\
\text { biopsy, surgical case number }\end{array}$ \\
$\begin{array}{l}\text { Primary and secondary } \\
\text { diagnoses }\end{array}$ & As per pathologists' discretion \\
Quantitative pathology details & $\begin{array}{l}\text { Number of glomeruli } \\
\text { Number of obsolete glomeruli }\end{array}$ \\
& $\begin{array}{l}\text { Number of segmentally sclerotic } \\
\text { glomeruliNumber of glomeruli with } \\
\text { Crescents } \\
\text { Severity of interstitial fibrosis and tubular } \\
\text { atrophy } \\
\text { Severity of arteriolar and arterial vascular } \\
\text { disease }\end{array}$ \\
O-3+ for each of lgG, IgM, IgA, C3 and C19 \\
Immunofluorescence findings nephropathy: MEST score \\
Tisease-specific classifications \\
Transplant allograft: Banff classification \\
Lupus nephritis: ISN/RPS classification \\
FSGS: Columbia classification \\
ANCA vasculitis: EUVAS classification \\
\hline
\end{tabular}

MEST = based on Oxford classification [12]. ISN/RPS = International Society of Nephrology / Renal Pathology Society. FSGS = focal segmental glomerulosclerosis. weight. While these variables are not extensive, they represent a balance between feasibility and cost, with the minimum data required to accurately describe a cohort of patients with GN. The same data is collected for all CKD, ESRD, and kidney transplant patients as part of the provincial mandate of the BCPRA.

\section{Table 3 List of native-kidney biopsy diagnoses that are used to identify patients eligible for registration in the BC GN Registry}

Histologic diagnoses enrolled in the BC GN registry

Alport's disease

Idiopathic nodular glomerulosclerosis

Amyloidosis: $\mathrm{AL}$

IgA nephropathy

Amyloidosis: AA

IgM nephropathy

Amyloidosis: other

Immunotactoid GN

Anti-GBM antibody

(Goodpasture's) GN

Autoimmune/connective tissue disease associated GN

Cryoglobulinemic GN

Light chain deposition disease

C3 GN

C1q nephropathy

Dense deposit disease

Light and heavy chain deposition disease

Lupus nephritis

Minimal change disease

Membranoproliferative GN, complement mediated

Membranoproliferative GN, IC mediated

Fabry's disease

Membranous nephropathy

Fibrillary GN

Mesangial proliferative GN

Focal segment

glomerulosclerosis

GN with monoclonal

immunoglobulin deposits

GN not otherwise specified

Pauci-immune (ANCA) GN

Post-infectious GN

Heavy chain deposition

Proliferative GN

disease

Thin basement membrane disease

Henoch-Schonlein purpura

Waldenstrom's macroglobulinemia

This list may be updated in the future as histologic classifications change over time. 
Table 4 The number of cases of each type of GN in the BC pathology databases from 2000 to 2012

\begin{tabular}{cc}
\hline GN type & Number of cases \\
\hline Minimal Change Disease & 263 \\
IgA Nephropathy & 827 \\
Focal Segmental Glomeruloslerosis & 846 \\
Membranous Nephropathy & 418 \\
Lupus Nephritis & 738 \\
ANCA Vasculitis & 381 \\
\hline
\end{tabular}

The average number of GN cases per year is 267 .

\section{Capture of renal outcomes and mortality}

There is complete capture of patient enrollment in CKD clinics, timing and type of ESRD modality, transplant outcomes and mortality as part of the provincial mandate and existing infrastructure. Only those patients who move out of province are lost to follow up. Therefore, these outcomes will be available for all patients in the BC GN Registry.

\section{Data security and integrity}

The BC GN Registry is contained within PROMIS, which is an administrative health care database that stores fully identified patient-level data and is managed by the BCPRA under regulation by the provincial government of $\mathrm{BC}$. It is a fully encrypted Oracle-based database, with regular backup to both servers and magnetic tapes. All users have access only to data deemed necessary for their clinical and/or administrative positions, and require secure passwords for login. All successful and unsuccessful login attempts and data entry events are tracked. The security systems and practices for PROMIS are similar to those used for other governmental health care agencies in $\mathrm{BC}$.

\section{Linkage of the BC GN Registry with other administrative databases}

Each person in $\mathrm{BC}$ has a unique Personal Health Number (PHN), which is required for registration into PROMIS and is therefore captured by the BC GN Registry at the time of kidney biopsy. Using the PHN, the BC GN Registry has been linked to various other governmental administrative databases. Examples include the BC Cardiac Registry to identify angiographic or surgical cardiac interventions; the $\mathrm{BC}$ Cancer Agency to identify malignancy events; PharmaNet to capture all medications dispensed at out-patient pharmacies in the province; the Canadian Institute of Health Information Discharge Abstract Database to identify hospital admissions, discharge diagnoses and comorbidities; the Medical Services Plan to identify all visits to physicians; and the Vital Statistics Database for cause and date of death. This is shown in Figure 1. Processes and procedures for this linkage will be in accordance with all privacy legislation, and differ depending on the purpose of data usage.

\section{Utility and discussion}

British Columbia is a Canadian province with over 4.6 million inhabitants and approximately 289 cases of GN per year. This represents a substantial incidence of patients who consume significant health care resources and are at increased risk of progression to ESRD. Prior to this initiative, as is the case in many other national and international jurisdictions, in $\mathrm{BC}$ there was no consistent formalized framework or data capture to inform the care of patients with GN. Thus, despite the large number of incident and prevalent GN patients, there has been little published data on short and long-term outcomes.

The BC GN Network and Registry represents a novel approach of integrating prospective data collection on GN patients into a provincial clinical care program for glomerular diseases, which will facilitate the development and evaluation of health policy and health services initiatives aimed at improving the outcomes of patients with GN. The BC GN Network is a provincial initiative that incorporates representation from BCPRA administrative bodies involved in health services delivery relevant to GN, one component of which is the GN Registry within PROMIS. This integration of data capture within the larger framework of GN health administration will allow for robust evaluation of health policy or clinical care programs developed by the BC GN Network. Indeed, this model has proven effective at improving the outcomes of all-cause CKD patients in BC. PROMIS was designed as a CKD administrative database to inform the renal-specific health care delivery of the BCPRA. It has been used to show that CKD patients managed in multi-disciplinary care clinics have better metabolic parameters and improved survival on dialysis compared to those managed by nephrologists [14]. Further, comprehensive data obtained from PROMIS has been used to identify substantial cost savings by managing CKD patients in a combined specialist clinic, describe provincial variability in testing for hepatitis $B$ immune status in renal patients, and identify rare but severe complications from dialyzer membranes [15-17]. The model of integrating data collection into health care delivery has already proven effective in $\mathrm{BC}$ in all-cause CKD, and it is anticipated that similar results will be seen in patients with glomerular disease.

Combining the BC GN Registry with the health services delivery and administrative activities of the BCPRA has the additional benefit of improving the scope and reducing the cost of data capture for a rare disease such as GN. Existing GN registries have provided substantial insight into the natural history of glomerular diseases and have been invaluable in the advancement of GN 
research [18-22]. However, most GN registries have been research-based, limited in the geographic scope or type of patient recruitment, and have relied on substantial amounts of manual data collection [1]. By comparison, the BC GN Registry is unique in that it is integrated into the existing provincial CKD health delivery infrastructure and uses linkages to laboratory and administrative databases to minimize laborious data entry, improving data quality and quantity. This approach ensures the long-term feasibility of the registry and allows complete capture of relevant patient information throughout the geographically diverse province of $\mathrm{BC}$. In the study of rare diseases such as GN, comprehensive data capture of large numbers of patients over long periods of time is viewed as essential.

Research in the field of GN has been substantially limited by the rarity of the disease and the poor availability and substantial cost of detailed registries with long-term follow-up that sample large source populations $[1,23]$. The BC GN Registry provides a feasible and sustainable solution that will produce a rich dataset with clinically relevant renal and mortality outcomes capable of supporting research in glomerular disease. Data collection leverages existing interfaces to automatically capture laboratory results and ESRD status, which limits manual data entry to only core elements. Further, the ability to link administrative datasets provides the opportunity to capture detailed outcome information as shown in Figure 1 without the need for laborious prospective data entry. The provincial scope of the BC GN Registry allows patient recruitment from the entire population of $\mathrm{BC}$, thereby improving generalizability compared to research-based $\mathrm{GN}$ registries that are often subject to selection bias from focused enrollment of patients from tertiary referral centres. The universal health care system in Canada helps reduce some of the bias that results from differential access to care. This approach to data collection ensures that over time the BC GN Registry will have a sufficient number of cases with detailed data capture to support clinical outcomes and health services research in GN. Furthermore, a coordinated provincial strategy for identifying and following patients with GN will facilitate recruitment into prospective clinical trials. This unique approach can serve as a model for other provinces in Canada, and as a nidus for Canadian GN collaboration.

There are several limitations to the data collected in the BC GN Registry. Firstly, in an attempt to improve the feasibility of data collection and the geographic distribution of patient enrolment, decisions were made regarding the need for a limited set of core clinical variables. This can be viewed as a relative limitation, as additional linkages with administrative datasets will be able to supplement prospectively collected data. Specific research questions can always be supplemented by review of individual patient records as required. Further, the BC GN Registry only enrolls patients with biopsy-proven disease, and would not necessarily be generalizable to patients with presumed GN that has not been confirmed on kidney biopsy. However, standard nephrology practice in Canada is to biopsy adult patients with clinically relevant proteinuria, especially in the context of declining renal function [24]. As such, patients with GN who do not undergo a biopsy would be expected to have mild disease with a favourable long-term prognosis. Further, the reliance on kidney biopsy as the point of identification and registration may complicate the ability to differentiate primary versus secondary disease in future research analyses. This will be partially mitigated by complete capture of laboratory data (such as autoimmune and viral serology) that are used to identify secondary causes of GN. Finally, there is a need for regular data validation methods so as to ensure correct classification and data entry. Pragmatic and robust processes of data validation are being developed and will be the subject of a future publication, once a sufficient sample of patient records is available.

\section{Conclusion}

The BC GN Network is a unique approach to improving the understanding and care of patients with $\mathrm{GN}$, a rare but important subset of patients with CKD. The BC GN Registry constitutes the core of the network, and is a comprehensive prospective database of patients with GN in $\mathrm{BC}$. The existence of the registry will support future clinical outcomes and health services research in glomerular disease. The GN network utilizes a novel approach to data collection in $\mathrm{GN}$ by being integrated into the existing CKD health services infrastructure and by including automatic capture of data through linkages to laboratories and administrative databases. This reduces laborious manual data entry, thereby broadening the geographic region from which patients can be recruited. The BC GN Network is a model of integrating knowledge acquisition, interpretation and application in real time.

\section{Availability and requirements}

The BC GN Registry data is contained in PROMIS, which is under regulation by governmental agencies in $\mathrm{BC}$ (the BCPRA and the Provincial Health Services Authority). Details about accessing PROMIS data for research purposes can be found at the BCPRA website (www.bcre nalagency.ca). Anyone interested in accessing PROMIS data for non-commercial research purposes must complete a data application request (which will be vetted by the BCPRA) and secure ethics approval through the University of British Columbia. Linkage of PROMIS data to other administrative databases may require additional approval from appropriate data stewards. 


\section{Additional file}

\section{Additional file 1: BC Glomerulonephritis (GN) Network Steering} Committee.

\section{Competing interest}

The authors declare that they have no competing interests.

\section{Authors' contributions}

$\mathrm{SB}, \mathrm{MB}, \mathrm{JG}$ and $\mathrm{AL}$ have been involved in the development and implementation of the BC GN Registry and the BC GN Network, including the logistics and processes around patient registration and data capture. All authors contributed to manuscript writing, editing and submission. All authors read and approved the final manuscript.

\section{Acknowledgements}

The authors would like to acknowledge the nephrologists in BC for their efforts in recruiting patients and collecting data for the BC GN Registry, and to the members of the BC GN Network Steering Committee.

\section{Author details}

'Division of Nephrology, University of British Columbia, Vancouver, BC, Canada. ${ }^{2}$ BC Provincial Renal Agency, Vancouver, BC, Canada. ${ }^{3}$ Centre for Health Evaluation and Outcomes Research, St. Paul's Hospital, Vancouver, BC, Canada. ${ }^{4}$ Division of Nephrology, University of Toronto, Toronto, BC, Canada.

Received: 14 August 2013 Accepted: 24 October 2013

Published: 29 October 2013

\section{References}

1. Leaf DE, Appel GB, Radhakrishnan J: Glomerular disease: why is there a dearth of high quality clinical trials? Kidney Int 2010, 78(4):337-342.

2. McGrogan A, Franssen CF, de Vries CS: The incidence of primary glomerulonephritis worldwide: a systematic review of the literature. Nephrol Dial Transplant 2011, 26(2):414-30.

3. Canadian Institute for Health Information: Annual Report-Treatment of EndStage Organ Failure in Canada, 2001 to 2010; 2012. https://secure.cihi.ca/ estore/productFamily.htm?locale=en\&pf=PFC1696.

4. Chou YH, Lien YC, Hu FC, Lin WC, Kao CC, Lai CF, et al: Clinical Outcomes and Predictors for ESRD and Mortality in Primary GN. Clin J Am SoC Nephrol 2012, 7(9):1401-8.

5. Troyanov S, Wall CA, Miller JA, Scholey JW, Cattran DC: Idiopathic membranous nephropathy: definition and relevance of a partial remission. Kidney Int 2004, 66(3):1199-205.

6. Troyanov S, Wall CA, Miller JA, Scholey JW, Cattran DC: Focal and segmental glomerulosclerosis: definition and relevance of a partial remission. J Am Soc Nephrol 2005, 16(4):1061-8.

7. Schieppati A, Mosconi L, Perna A, Mecca G, Bertani T, Garattini S, et al: Prognosis of untreated patients with idiopathic membranous nephropathy. N Engl J Med 1993, 329(2):85-9.

8. Reich HN, Troyanov S, Scholey JW, Cattran DC: Remission of proteinuria improves prognosis in IgA nephropathy. J Am Soc Nephrol 2007, 18(12):3177-83.

9. Cattran DC, Rao P: Long-term outcome in children and adults with classic focal segmental glomerulosclerosis. Am J Kidney Dis 1998, 32(1):72-9.

10. Pesce F, Schena FP: Worldwide distribution of glomerular diseases: the role of renal biopsy registries. Nephrol Dial Transplant 2010, 25(2):334-6.

11. Barbour S, Er L, Magil A, Reich H, Levin A: Ethnic differences in renal and patient survival in IgA nephropathy. Abstract at the World Congress of Nephrology; 2011

12. Cattran DC, Coppo R, Cook HT, Feehally J, Roberts IS, Troyanov S, et al: The Oxford classification of IgA nephropathy: rationale, clinicopathological correlations, and classification. Kidney Int 2009, 76(5):534-45.

13. Komenda $P$, Beaulieu M, Seccombe D, Levin A: Regional implementation of creatinine measurement standardization. J Am Soc Nephrol 2008, 19(1):164-9.

14. Curtis BM, Ravani P, Malberti F, Kennett F, Taylor PA, Djurdjev O, et al: The short- and long-term impact of multi-disciplinary clinics in addition to standard nephrology care on patient outcomes. Nephrol Dial Transplant 2005, 20(1):147-54
15. Weber C, Beaulieu M, Djurdjev O, Er L, Taylor P, Ignaszewski A, et al: Towards rational approaches of health care utilization in complex patients: an exploratory randomized trial comparing a novel combined clinic to multiple specialty clinics in patients with renal diseasecardiovascular disease-diabetes. Nephrol Dial Transplant 2012, 27(3):iii104-110.

16. Beaulieu M, Krajden M, Buxton J, Er L, Djurdjev O, Levin A: Variability of hepatitis $B$ testing in British Columbian ESRD patients: the case to focus on implementation of guidelines. Am J Kidney Dis 2008, 52(5):939-46.

17. Kiaii M, Djurdjev O, Farah M, Levin A, Jung B, MacRae J: Use of electronbeam sterilized hemodialysis membranes and risk of thrombocytopenia. JAMA 2011, 306(15):1679-87.

18. Regional program for the study of glomerulonephritis: Central Committee of the Toronto Glomerulonephritis Registry. Can Med Assoc J 1981 124(2):158-61

19. Schena FP, Cerullo G, Torres DD, Scolari F, Foramitti M, Amoroso A, et al: The IgA nephropathy Biobank. An important starting point for the genetic dissection of a complex trait. BMC Nephrol 2005, 6:14.

20. Rivera F, Lopez-Gomez JM, Perez-Garcia R: Clinicopathologic correlations of renal pathology in Spain. Kidney Int 2004, 66(3):898-904.

21. Rivera F, Lopez-Gomez JM, Perez-Garcia R: Frequency of renal pathology in Spain 1994-1999. Nephrol Dial Transplant 2002, 17(9):1594-602.

22. Li LS, Liu ZH: Epidemiologic data of renal diseases from a single unit in China: analysis based on 13,519 renal biopsies. Kidney Int 2004, 66(3):920-3

23. KDIGO Clinical Practice Guidelines for Glomerulonephritis. Kidney Int 2012, S2(2):139-274.

24. Fuiano G, Mazza G, Comi N, Caglioti A, De Nicola L, lodice C, et al: Current indications for renal biopsy: a questionnaire-based survey. Am J Kidney Dis 2000, 35(3):448-57.

doi:10.1186/1471-2369-14-236

Cite this article as: Barbour et al: An overview of the British Columbia Glomerulonephritis network and registry: integrating knowledge generation and translation within a single framework. BMC Nephrology $201314: 236$.

\section{Submit your next manuscript to BioMed Central and take full advantage of:}

- Convenient online submission

- Thorough peer review

- No space constraints or color figure charges

- Immediate publication on acceptance

- Inclusion in PubMed, CAS, Scopus and Google Scholar

- Research which is freely available for redistribution 